\title{
A study on Nonlinear Programming Problem in Fuzzy Environment
}

\author{
M. Lalitha, ${ }^{1}$, Dr. C. Loganathan, ${ }^{2}$ \\ ${ }^{I}$ Department of Mathematics,Kongu Arts and Science College,Erode. \\ ${ }^{2}$ Principal,Maharaja Arts and Science College, Coimbatore.
}

\begin{abstract}
In that paper, the fuzzy nonlinear programming problem is discussed. In order to obtain more accurate solution, the properties of fuzzy set and fuzzy number with linear membership function and fuzzy maximum decision maker is utilized to fuzzifying the crisp problem. In the illustration, we have given a refinement to obtain this solution.
\end{abstract}

Keywords: Fuzzy numbers, Nonlinear programming, Optimization problem, Fuzzy maximum decision making.

\section{Introduction}

Fuzzy nonlinear programming problem (FNLPP) is useful in solving problems which are difficult, impossible to solve due to the imprecise, subjective nature of the problem formulation or have an accurate solution. In this paper, we will discuss the concepts of fuzzy decision making introduced by [1] and the maximum decision [15] that is used in NLPP to find the optimal decision (solution). This decision making used in fuzzy linear programming problem [8] and [7]. Furthermore, this problems has fuzzy objective function and fuzzy variables in the constraints [13], [10], and [5], where the fuzzy left and right hand side coefficients on constraints [14]. In addition, the fuzzy NLPP is used in quadratic programming [9,11] which has fuzzy multiobjective function and fuzzy parameters on constraints so in our NLPP that have fuzzy properties on the inequality $(\tilde{\leq}, \tilde{z})$ and have fuzzy linear membership function. The outline of this paper is as follows: In this section 1 , we state in section 2 , the earlier method is briefly explained. In section 3 , we give our refinement for the same problem by extending it to a general setup of nonlinear programming problem of this type.

\section{Preliminary}

In this section, several necessary basic concepts are recalled.

Definition 2.1: [15] If $\mathrm{x}$ is a collection of objects denoted generally by $X$, then a fuzzy set $\tilde{\mathrm{A}}$ in $X$ is a set of order pairs

$$
\tilde{\mathrm{A}}=\left\{\left(x, \mu_{\tilde{\mathrm{A}}}\right): x \in X\right.
$$

Where $\mu_{\tilde{A}}: x \rightarrow[0,1]$ is called the membership function or grade of membership of $x$ in Ãwhich maps $\mathrm{x}$ to the membership range $M$ (when $M$ contains only the two points 0 and 1), Âis non fuzzy and $\mu_{\tilde{\AA}}$ is identical to the characteristic function of crisp set. It should be emphasized that the range of membership function is a subset of the non-negative real numbers. The elements with a zero degree of membership are normally not listed.

Definition 2.2: [2] The function $L: X \rightarrow[0,1]$ is a function with two parameters defined as:

$$
\mathrm{L}(\mathrm{x} ; \alpha, \beta)=\left\{\begin{array}{c}
1, \\
\frac{\alpha+\mathbb{\beta}-x}{\mathrm{~B}} \\
0,
\end{array}\right.
$$

$$
\begin{gathered}
\text { if } x<\alpha \\
\text { if } \alpha<x<\alpha+\mathrm{B} \\
\text { if } x>\beta
\end{gathered}
$$

It is called the trapezoidal linear membership function. This type of fuzzy number is very useful which has a large non convex fuzzy rejoin set. Clearly, it gives us the high degree of $\mu_{\tilde{A}}$. (See the Fig. 1). 
Definition 2.3: [1] Given a fuzzy goal (fuzzy objective function) $\tilde{\mathrm{G}}$ and fuzzy constraints $\tilde{\mathrm{C}}$ in a space of alternatives $\mathrm{X}$. The $\tilde{\mathrm{G}}$ and $\tilde{\mathrm{C}}$ combine to form a decision, $\tilde{\mathrm{D}}$, which is a fuzzy set resulting from intersection of $\tilde{\mathrm{G}}$ and $\tilde{\mathrm{C}}$.

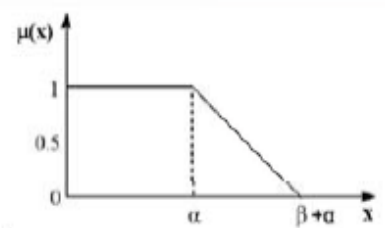

Figure 1: L-function

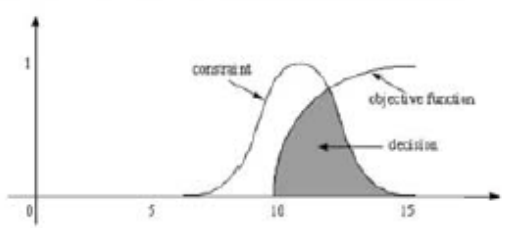

Figure 2: Fuzzy decision

Furthermore, $\tilde{D}=\tilde{\mathrm{G}} \bigcap \tilde{\mathrm{C}}$ is the membership function of $\tilde{\mathrm{D}}$ can be defined as $\mu_{\tilde{\mathrm{D}}}=\min \left\{\mu_{\tilde{\mathrm{G}}}, \mu_{\tilde{\mathrm{C}}}\right\}$. In general, if we have $n$ goals $\tilde{\mathrm{G}}_{1}, \ldots, \tilde{\mathrm{G}}_{\mathrm{n}}$ and $\mathrm{m}$ constraints $\tilde{\mathrm{C}}_{1}, \ldots, \tilde{\mathrm{C}}_{\mathrm{m}}$, then, the resultant decision can be defined as

$\tilde{\mathrm{D}}=\tilde{\mathrm{G}}_{1} \cap \ldots \cap \tilde{\mathrm{G}}_{\mathrm{n}} \cap \tilde{C}_{1} \cap \ldots \cap \tilde{C}_{\mathrm{m}}$

Therefore, for $j=1,2, \ldots$, nand $i=1,2, \ldots$, mit can be written as follows

$\mu_{\tilde{\mathrm{D}}}=\min \left\{\min \left\{\mu_{\tilde{\mathrm{G}} \mathrm{j}}\right\}, \min \left\{\mu_{\tilde{\mathrm{C}} \mathrm{i}}\right\}\right\}$

$=\min \left\{\mu_{\tilde{\mathrm{G}} 1}, \ldots, \mu_{\tilde{\mathrm{G}} \mathrm{n}}, \mu_{\tilde{\mathrm{C}} 1}, \ldots, \mu_{\tilde{\mathrm{C}} \mathrm{m}}\right\}$

$=\min \left\{\mu_{\tilde{\mathrm{G}} \mathrm{j}}, \ldots, \mu_{\tilde{\mathrm{C}} \mathrm{i}}\right\}$

(See the Fig. 2)

Remarks: If the decision-maker wants to have "crisp" decision proposal, it is appropriate to suggest which of them have the highest degree of membership in the fuzzy set "decision." The maximizing decision is defined by

$\mathrm{X}_{\max }=\max \mathrm{M}_{\tilde{\mathrm{D}}}(X)=\max \left\{\mu_{\tilde{\mathrm{D}}} \mathrm{j}(X), \mu_{\tilde{\mathrm{Ci}}}(X)\right\}$

$x x$

where $\tilde{D}_{j}$ and $\tilde{\mathrm{C}}_{\mathrm{i}}$ are in Definition 2.3, for $\mathrm{i}=1,2, \ldots, \mathrm{m}, \mathrm{j}=1,2, \ldots, \mathrm{n}[15]$.

\section{Fuzzy nonlinear programming problems}

In this section, we discuss the optimization problem with nonlinear fuzzy objective function and fuzzy flexible nonlinear constraints. Consider the following nonlinear programming problem:

Subject to

$$
\operatorname{Min} / \operatorname{Max} \mathrm{f}(x)
$$

$$
\mathrm{g}_{\mathrm{i}} \quad(x) \geq(\leq) \mathrm{b}_{\mathrm{i}}, \quad \mathrm{i}=1,2, \ldots, \mathrm{m} .
$$

For all $x \in \mathrm{R}^{\mathrm{n}}$ and $x \geq 0$. The fuzzy version of the problem (3.1) is

Subject to

$$
\left.\operatorname{Min} / \operatorname{Max} \mathrm{f}^{\boldsymbol{X}}\right)
$$

$$
\mathrm{g}_{\mathrm{i}}(x) \tilde{\geq}(\tilde{\leq}) \mathrm{b}_{\mathrm{i}}, \quad \mathrm{i}=1,2, \ldots, \mathrm{m} .
$$

For all $x \in \mathrm{R}^{\mathrm{n}}$ and $x \geq 0$. In problem (3.2), the tilde sign denotes a fuzzy satisfaction of the constraints. It is clear that these constraints are flexible constrains. The fuzzy max (min) corresponds to achieving the highest (lowest) possible aspiration level for the general $\mathrm{f}(\mathcal{X})$. This problem can be solved by using the properties of fuzzy decision making problems (3.1) and (3.2) as follows: 
Fuzzify the objective function by calculating the lower and the upper bounds of the optimal values. The bounds of optimal values $z_{1}$ and $z_{\mathrm{u}}$ can be obtained by solving the standard crisp NLPP as follows:

Subject to

$$
\mathrm{Z}_{\mathrm{l}}=\operatorname{Min} / \operatorname{Max} \mathrm{f}(\boldsymbol{x})
$$

$$
\mathrm{g}_{\mathrm{i}}(\boldsymbol{X}) \geq(\leq) \mathrm{b}_{\mathrm{i}}, \quad \mathrm{i}=1,2, \ldots, \mathrm{m} .
$$

For all $x \in \mathrm{R}^{\mathrm{n}}$ and $x \geq 0$ and

Subject to

$$
\mathrm{z}_{2}=\operatorname{Min} / \operatorname{Max} \mathrm{f}(\boldsymbol{x})
$$

$$
\mathrm{g}_{\mathrm{i}}(\boldsymbol{X}) \geq(\leq) \mathrm{b}_{\mathrm{i}}+\mathrm{p}_{\mathrm{i}}, \quad \mathrm{i}=1,2, \ldots, \mathrm{m} .
$$

For all $\mathrm{x} \in \mathrm{R}^{\mathrm{n}}$ and $x \geq 0$. Where the objective function take the values between $\mathrm{z}_{1}$ and $\mathrm{z}_{2}$. Let $\mathrm{z}_{\mathrm{l}}=\min \left(\mathrm{z}_{1}\right.$, $\left.z_{2}\right)$ and $z_{u}=\max \left(z_{1}, z_{2}\right)$, where $z_{1}$ and $z_{u}$ are the lower and upper bounds of the optimal values. Suppose $\tilde{M}$ is the fuzzy set representing the objective function $\mathrm{f}(\mathcal{X})$ such that $\tilde{\mathrm{M}}=\left\{\left({ }^{X}, \mu_{\tilde{\mathrm{M}}}(\mathcal{X})\right):{ }^{X} \in \mathrm{R}^{\mathrm{n}}\right\}$,

$\mu_{\tilde{\mathrm{M}}}(x)_{=}\left\{\begin{array}{cl}1, & \text { if } z_{u}<f(x) \\ \frac{f(x)-z_{l}}{z_{u}-z_{l}} & \text { if } z_{l}<f(x)<z_{u} \\ 0, & \text { if } z_{l}>f(x)\end{array}\right.$.

\section{Numerical Example:}

In the following example, we will illustrate presented theory. Suppose we are going to make a box for airport shipping [3]. The box is made of two materials; the top of the box is made of a material costing $\$ 17$ per square foot, and the rest of the box is 0made of material that cost $\$ 3$ per square foot. Then, the baggage restrictions require that the dimensions of the box end of it must sum to at most 3 foot. The dimensions of the box of should be a maximum volume costing and not more than $\$ 108$. Mathematical description $x_{1}, x_{2}$ and $x_{3}$ are present the length,

width and height of the box respectively. The NLP optimization problem is

$\operatorname{Max}^{x} x_{2} x_{3}=\mathrm{z}$

Subject to

$\mathrm{g}_{1}: x_{2}+x_{3} \leq 3$

$$
\mathrm{g}_{2}: 17^{x_{1} x_{2}}+3\left(x_{1} x_{2}+2 x_{1} x_{2}+2 x_{2} x_{3}\right) \leq 108
$$

$x_{1}, x_{2}, x_{3} \geq 0$

The optimal solution is $X \quad *_{1}=2.99526551, x \quad *_{1}=1.00316204$, and $X *_{1}=1.99687905$. Therefore $\mathrm{Z}^{*}=5.99996738$ satisfies the constraints of problem (4.1). Now, the fuzzy version of the problem is

$$
\begin{array}{llllll}
\text { Max } & x & 1^{x} & 2^{x} & 3 & = \\
\end{array}
$$

Subject to

$\mathrm{g}_{1}: x_{2}+x_{3} \tilde{\leq} 3$

$$
\mathrm{g}_{2}: 17 x_{1} x_{\mathbf{2}}+3\left(x_{\mathbf{1}} x_{\mathbf{2}}+2 x_{\mathbf{1}} x_{\mathbf{2}}+2 x_{\mathbf{2}} x_{\mathbf{3}}\right) \tilde{\leq} 108
$$

$x_{1}, x_{2}, x_{3} \geq 0$

Therefore, $b_{1}=3$ and $b_{2}=108$. In order to obtain $p_{1}$ and $p_{2}$, we have $\tilde{3}=\left\{\left(x, \mu_{\tilde{3}}(x)\right): x^{X} \in \mathbf{R}^{\mathrm{n}}\right\}$

Where

$$
\mu_{3}(x)=\left\{\begin{array}{cc}
1, & \text { if } x<3 \\
\frac{5-x}{2} & \text { if } 3 \leq x \leq 5 \\
0, & \text { if } x \geq 5
\end{array}\right.
$$


(4.3)

It is shown in the Figure (4). Hence $\mathrm{p}_{1}=2$. Similarly $\mathrm{p}_{2}$ canbe obtained by

圆 EMBED Equation.3 固圆圆 $\quad=\left\{\left(\begin{array}{llll}x & \mu_{10 \tilde{8}} & (x)) & : x \in \mathbf{R}^{n}\end{array}\right\}\right.$

Where

$$
\mu_{10 \tilde{8}}(x) \quad\left\{\begin{array}{cc}
1, & \text { if } x<108 \\
\frac{120-x}{12} & \text { if } 108 \leq x \leq 120 \\
0, & \text { if } x \geq 108
\end{array}\right.
$$

We get $\mathrm{p}_{2}=12$. It is shown in the Figure (5). Now, we can find $z_{l}$ and $z_{u}$ by solving the two crisp NLPP as follows:

1. $\mathrm{z}_{1}=\mathrm{z}$. Since the problem is the same first problem, and they have the same solution, therfore $\mathrm{z}_{1}=5.99996738 \$$.

2. $\mathrm{z}_{2}=\operatorname{Max}^{x} 1_{1}^{x} 2^{x}{ }_{3}$

Subject to

$x \quad{ }_{1}^{*}=1.77424067, \quad x_{2}{ }^{*}=1.44655028, \quad x_{3}{ }^{*}=3.55344549$.

which is satisfying the constraints. Finally, $\mathrm{z}_{2}=9.12001855$. Let $\tilde{M}$ be the fuzzy set of all objective function such that

$\tilde{\mathrm{M}}=\left\{\left({ }^{x}, \mu_{\tilde{\mathrm{M}}}(x)\right): x \in \mathbf{R}\right\}$

And

$\mu_{\hat{M}}(x)=\left\{\begin{array}{cl}1, & \text { if } 5.999987738<x_{\mathbf{1}} x_{\mathbf{2}} x_{\mathbf{3}} \\ \frac{x_{\mathbf{1}} x_{\mathbf{2}} x_{\mathbf{3}}-5.999987738}{9.12001855-5.99987738} & \text { if } 5.999987738 \div x_{\mathbf{1}} x_{\mathbf{2}} x_{\mathbf{3}}<9.1200 \\ \mathbf{0} & \text { if } x_{\mathbf{1}} x_{\mathbf{2}} x_{\mathbf{3}}<9.1200\end{array}\right.$

In addition, let $\tilde{\mathrm{C}}_{1}$ be the fuzzy set for $\mathrm{g}_{1}$ such that

$\tilde{\mathrm{C}}_{1}=\left\{\left({ }^{x}, \mu_{\tilde{\mathrm{c}} 1}(x)\right): x \in \mathbf{R}\right\}$

Where

$$
\mu_{\tilde{\mathrm{c}}}(x)=\left\{\begin{array}{cl}
1, & \text { if } x_{2}+x_{3}<3 \\
\frac{5-\llbracket\left(x \rrbracket_{2}+x_{3}\right)}{2} & \text { if } 3<x_{2}+x_{3}<5 \\
0, & \text { if } 5<x_{2}+x_{3}
\end{array}\right.
$$

and $\tilde{\mathrm{C}}_{2}$ be the fuzzy set for $\mathrm{g}_{2}$ such that

Where

$$
\tilde{\mathrm{C}}_{2}=\left\{\left(x, \mu_{\tilde{\mathrm{c}} 2}(x)\right): x \in \mathbf{R}\right\}
$$

$$
\mu_{\tilde{2} 2}(x)=(4.7)
$$

The fuzzy decision making for this problem is

$$
\mu_{\tilde{\mathrm{D}}}(x)=\min \left\{\mu_{\tilde{\mathrm{M}}}(x), \min \left\{\mu_{\tilde{\mathrm{C}} 1}(x) \ldots, \mu_{\tilde{\mathrm{C}} 2}\right\}\right\}
$$

for $\lambda=\min \left\{\mu_{\tilde{\mathrm{M}}}(x), \min \left\{\mu_{\tilde{\mathrm{C}} 1}(x), \mu_{\tilde{\mathrm{C}} 2}(x)\right\}\right\}$, with optimal decision $x^{*}=\operatorname{Max} \lambda$.

$x_{1}{ }^{*}=1.5, \quad x_{2}{ }^{*}=2.5, \quad x_{3}{ }^{*}=1.5, \quad \operatorname{Max} \mathrm{Z}=5.625$

Where $x_{1}, x_{2}, x_{3} \geq 0$ and $0 \leq \lambda \leq 1$

\section{Data Algorithm Analysis and Results}

In the above output, we give an extenstion to proceed further taking a trial solution near to the exact solution. The resignment is given by the method.

$\mathbf{m}(\mathbf{k}+\mathbf{1})=\mathbf{m}(\mathbf{k}+(\mathbf{n}-\mathbf{1}) \mathbf{d})$ 
where $\mathrm{n}$ is the iteration number and $\mathrm{d}$ is the increment, $\mathrm{K}$ is the trial solution stage.

$x_{1}=x_{1}+.25$

$x_{2}=x_{2}-.25$

$x_{3}=x_{3}+.25$

\begin{tabular}{|l|l|l|l|l|l|l|l|}
\hline \multicolumn{1}{|c|}{$x_{1}$} & \multicolumn{1}{|c|}{$x_{2}$} & \multicolumn{1}{|c|}{$x_{3}$} & \multicolumn{1}{|c|}{$\mathrm{C}_{1}(\mathrm{x})$} & $\mathrm{C}_{2}$ & $\mathrm{D}(x)$ & $\mathrm{Z}$ \\
\hline 1.75 & 2.25 & 1.75 & 0.2854578418 & 0.50 & 8.2500 & 0.2854578418 & 6.890625 \\
\hline 2.0 & 2.0 & 2.0 & 0.6410232406 & 0.5 & 8.33333333 & 0.5 & 8.0 \\
\hline 2.25 & 1.75 & 2.25 & 0.9164612256 & 0.50 & 8.5000 & 0.5 & 8.859375 \\
\hline 2.50 & 1.50 & 2.50 & 1.0817240166 & 0.5 & 8.75 & 0.5 & 9.375 \\
\hline
\end{tabular}

Where $x_{1}, x_{2}, x_{3} \geq 0$ and $0 \leq \lambda \leq 1$. Therefore, the solution of problem is

$x_{1}{ }^{*}=1.75, \quad x_{2}{ }^{*}=2.25, \quad x_{3}{ }^{*}=1.75$

and $\lambda=0.2854578418$ which satisfy in the constraints while the result of crisp problem before fuzziness is

$x_{1} *=2.99526551, x_{2} *=1.00316204$, and $x_{3} *=1.99687905$

Now ,we can submit $x_{1} *, x_{2} *$ and $x_{3}$ in the objective function of the crisp NLP.

It can be obtained $z_{A F}=x_{1} * X_{2} * x_{3} *=6.890625$

Where $z_{l}<z_{A F}<z_{u}$.Clearly, in comparison the crisp problem we have more accurate solution.

\section{Conclusion}

We conclude that the above refinement gives a better result $t$ and the fuzzy environment is dealt within the arithmetic form of iteration.

\section{References}

[1] R.E. Bellman and L.A. Zadeh, Decision-making in a fuzzy environment. Management Sci, Vol. 17, No. 4 (1970) pp. 141-164.

[2] D. Drinkov, H. Hellendoorn, and M. Reinfrank, An Introduction to Fuzzy Control, Springer, Berlin, etc., 1993.

[3] Iyengar. P, Non-Linear Programming; Introduction, IEOR, Handout 19, 16 October 2002.

[4] Z. A. Kanaya , An Interactive Method for Fuzzy Multi objective Nonlinear Programming Problems, JKAU Sci., Vol. 22, No. 1 (2010) pp. 103-112.

[5] B. Kheirfam F. Hasani. Sensitivity analysis for fuzzy linear Programming problems with Fuzzy variables, Advanced Model and Optimization, Vol. 12, No. 2, 2010

[6] N. Mahdavi-Amiri and S.H. Nasseri, Duality in fuzzy variable linear programming, Transactions on Engineering, Computing and Technology, Vol. 6 (2005) pp. 115-118.

[7] S.H. Nasseri, A new method for solving fuzzy linear programming problems by solving linear programming, Applied Mathematical Sciences Journal, Vol. 50 (2008) pp. 2473-2480.

[8] S.H. Nasseri, E. Ardil, A. Yazdani and R. Zaefarian, Simplex method for solving linear programming problems with fuzzy numbers, Transactions on Engineering, Computing and Technology, Vol. 10 (2005) pp. 284-288.

[9] S.H. Nasseri, Fuzzy nonlinear optimization, The Journal of Nonlinear Analysis and its Applications, Vol. 1, No. 4 (2008) pp. 230235.

[10] V. Pandian, R. Nagarajan, and S. Yaacob, Fuzzy Linear Programming: A Modern Tool for Decision Making, JurnalTeknologi, Vol. 37 (2002) pp. 31-44.

[11] N. Ravi Shankar 1, G. AnandaRao, J. MadhuLatha and V. Sireesha, Solving a Fuzzy Nonlinear Optimization Problem by Genetic Algorithm, Int. J. Contemp. Math. Sciences, Vol. 5, No. 16, (2010) pp. $791-803$.

[12] E. K. Saadettin, K. D. Sanchoy, and Z. Gungor, A Mixed Integer Programming Formulation For Scheduling Of Virtual Manufacturing Cells (VCMS), International Journal Of Advance Manufacturing Technology, Vol. 2, No. 5 (2010) pp. 82-85.

[13] H. C. Wu, Duality Theory in Fuzzy Optimization Problems, Fuzzy Optimization and Decision Making, Vol. 3, (2004) pp. $345-365$.

[14] M. Yenilmez, N. Rafail, and K. Gasimor, Solving Fuzzy Liner Programming Problems with Linear Membership Function, Turk H. Math, TUBITAK, No. 26, (2002) pp. 375-369.

[15] H.J. Zimmermann. Fuzzy Sets, Decision Making, and Expert Systems, Kluwer-Nijho Publishing, Boston (1987). 